\title{
Crawling on the Heart: A Mobile Robotic Device for Minimally Invasive Cardiac Interventions
}

\author{
Nicholas A. Patronik ${ }^{1}$, Marco A. Zenati ${ }^{2}$, and Cameron N. Riviere ${ }^{1}$ \\ ${ }^{1}$ The Robotics Institute, Carnegie Mellon University, Pittsburgh, PA, USA \\ ${ }^{2}$ Division of Cardiothoracic Surgery, University of Pittsburgh, Pittsburgh, PA, USA
}

\begin{abstract}
This paper describes the development and preliminary testing of a robotic device to facilitate minimally invasive beating-heart intrapericardial interventions. We propose the concept of a subxiphoid-inserted mobile robot (HeartLander) with the ability to adhere to the epicardium, navigate to any location, and administer therapy under physician control. As compared to current laparoscopic cardiac surgical techniques, this approach obviates cardiac stabilization and eliminates access limitations. Additionally, it does not require lung deflation and differential lung ventilation, and thus could open the way to outpatient cardiac therapies. The current HeartLander prototype uses suction to maintain prehension of the epicardium and wire actuation to perform locomotion. A fiber optic videoscope displays visual feedback to the physician, who controls the device through a joystick interface. A working channel provides access for the insertion of various therapeutic tools. This prototype has demonstrated successful prehension and walking during open-chest beating-heart porcine trials.
\end{abstract}

\section{Introduction}

Minimally invasive cardiac surgery has become a major objective of the field due to the desire to avoid the morbidity associated with median sternotomy and cardiopulmonary bypass [1]. Sternotomy can be obviated by endoscopy. There are many cardiac surgical procedures that could conceivably be performed endoscopically, but in most cases the necessary instrumentation does not yet exist. The obstacles include not only miniaturization for endoscopic application, but also gaining access to certain hard-to-reach parts of the heart. Current instrumentation generally relies on rigid endoscopes, which can only reach a limited area on the epicardial surface from a given incision [2]. The multi-arm robot systems that are commercially available (at prices around US $\$ 1,000,000$ ) provide much of the needed dexterity for the realization of endoscopic heart surgery, but the problem of access remains unresolved for certain areas, such as the posterior wall of the left ventricle [2].

The challenges of minimally invasive access are further complicated by the goal of avoiding cardiopulmonary bypass. This requires surgery on the beating heart, greatly increasing the difficulties involved in worksite access and precise manipulation [3]. Instrumentation is needed that can provide stable manipulation of an arbitrary 
location on the epicardium while the heart is beating [4]. Thus far, progress in minimally invasive beating-heart surgery has been hindered by the need for endoscopic immobilization of the beating heart [2]. In open-heart surgery, immobilization is frequently accomplished using mechanical stabilizers such as the Acrobat (Guidant, Santa Clara, CA), TR ${ }^{3}$ IPOD (Chase Medical, Richardson, TX), and Octopus (Medtronic, Minneapolis, MN), which grasp a portion of the epicardial surface and hold it steady. Some endoscopic versions of such devices have now been developed. However, the resulting forces exerted on the myocardium can cause changes in the electrophysiological and hemodynamic performance of the heart, and care must be taken to avoid hemodynamic impairment or life-threatening arrhythmia [5]. As an alternative, several researchers in surgical robotics are investigating active compensation of heartbeat motion by visually tracking the epicardium and servoing the tool tips accordingly [6]. Such an approach, however, requires considerable expense for high-bandwidth actuation to manipulate in at least three degrees of freedom over a relatively large workspace [6].

All of these solutions address a problem that exists only because the tools are held by a robot (or surgeon) that is fixed to the table or the floor. We have taken a different approach: rather than trying to immobilize the heart surface to stabilize it in the (fixed) frame of reference of a table-mounted robotic device, the endoscopic device is mounted in the (moving) reference frame of the beating heart. This is accomplished using HeartLander, an innovative miniature robotic device that enters the pericardium through a minimally invasive port, attaches itself to the epicardial surface, then travels under its own power to the desired location and establishes a stable platform for surgery (Fig. 1). The problem of the beating-heart motion is thus avoided by attaching the device directly to the epicardium, and the problem of access is resolved by incorporating the capability for locomotion.

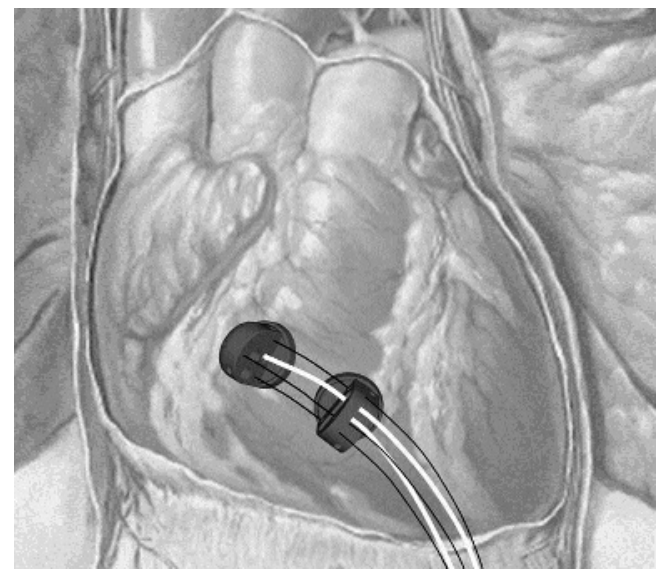

Fig. 1. Illustration of the HeartLander concept. The device is introduced using a subxiphoid approach, adheres to the epicardial surface, travels to the desired operative site, and administers the therapy under the control of the physician with video feedback 
Improved access and precise manipulation are not the only benefits of this approach. Port access for minimally invasive cardiac surgery has typically been transthoracic, primarily in order to accommodate the rigid endoscopes generally used for both manual and robot-assisted procedures. Transthoracic access to the heart requires the deflation of the left lung, which necessitates general endotracheal anesthesia and differential lung ventilation. But among both established procedures and innovative ones there is a considerable number that conceivably could be performed entirely within the pericardium; i.e., they do not intrinsically require access to the pleural space or anywhere else outside the pericardium. Examples include, but are not limited to:

- cell transplantation [7];

- $\quad$ gene therapy for angiogenesis [8];

- $\quad$ epicardial electrode placement for resynchronization [9];

- epicardial atrial ablation [10];

- $\quad$ intrapericardial drug delivery [11];

- $\quad$ ventricle-to-coronary artery bypass (VCAB) [12].

Minimally invasive instruments are not currently available for most of these procedures, and those that do exist are typically designed for transthoracic access. However, all of these procedures could be performed without deflating a lung if suitable instrumentation were available.

The ability of HeartLander to move to any desired location on the epicardium from any starting point enables minimally invasive cardiac surgery to be independent of the location of the pericardial incision. HeartLander can therefore be introduced via transpericardial rather than transthoracic access, through an incision below the xiphoid process. This subxiphoid transpericardial approach not only obviates sternotomy and cardiopulmonary bypass, but avoids entering the pleural space altogether. As a result, deflation of the left lung is no longer needed and it becomes feasible to use local or regional rather than general anesthetic techniques. This has the potential to open the way to ambulatory outpatient cardiac surgery.

A HeartLander prototype has been constructed and preliminary tests have been performed, including locomotion on exposed beating porcine hearts. This paper describes the design of the device and the results obtained from testing.

\section{Design}

Under direct control of the physician, HeartLander will facilitate cardiac interventions by attaching directly to the epicardial surface, crawling to any desired location, and administering the therapy. The current prototype consists of a distal drive mechanism and proximal support system, connected through a 1-m long tether. The drive mechanism is the miniature mobile portion of the robot that enters the patient and performs the aforementioned functions. The support system is the large stationary portion that is externally located and contains all active components that control the drive mechanism. These include the motors for actuation, the pump to supply vacuum pressure, and the PC for visual feedback and control. The tether transmits the functionality of 
the support system to the drive mechanism. This tethered design allows the therapeutic portion of the robot (drive mechanism and tether) to be passive, lightweight, inexpensive, and largely disposable.
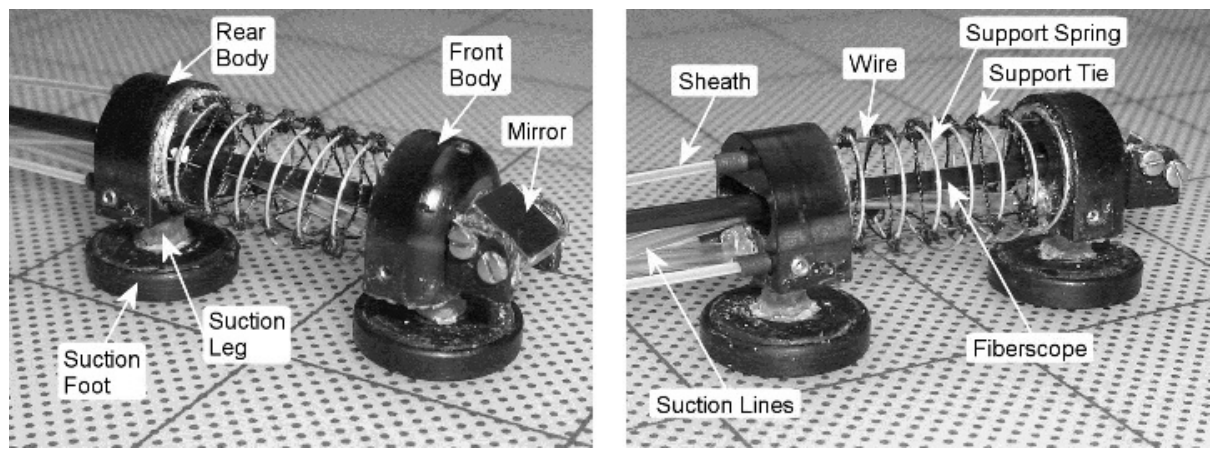

Fig. 2. The drive mechanism of the wire-actuated HeartLander prototype (lines mark a 25.4millimeter grid and the dots are spaced 2 millimeters apart)

At the start of the procedure, HeartLander will be placed directly on the epicardial surface, beneath the pericardium, using a rigid endoscopic tool specifically designed for this purpose. This instrument will be introduced into the thoracic cavity through an incision made just below the xiphoid process of the sternum. The front and back modules of the drive mechanism are each $16 \mathrm{~mm}$ tall and have circular footprints $13 \mathrm{~mm}$ in diameter, thus allowing the device to pass through a $20-\mathrm{mm}$ cannula. Once the treatment is complete, HeartLander will be retrieved by manually retracting the tether back through the endoscope. This also serves as the recovery method should the device become dislodged during the procedure.

The drive mechanism adheres to the epicardium using suction. Suction has proven to be effective for epicardial prehension in surgical stabilizers such as the Octopus $^{\mathrm{TM}}$ and Starfish ${ }^{\mathrm{TM}}$ (Medtronic, Minneapolis, MN), as well as general prehension in mobile robotics [13]. The suction forces are applied by two independent suction feet that are attached by compliant legs beneath the front and rear body sections of the drive mechanism. The vacuum pressure is supplied to the feet by the external support system pump through two vacuum lines that pass through the tether (Fig. 2). The pump provides a vacuum pressure of $-0.08 \mathrm{~N} / \mathrm{mm}^{2}$, which was found to be effective and safe for use in FDA-approved cardiac stabilizers. The suction forces generated by this pressure have proven effective for our application as well, and did not damage the epicardial tissue (see Sect. 3). The compliant legs allow the suction feet to conform to the curvature of the epicardial surface during locomotion. Fluids and small biological particles drawn through the suction feet and vacuum lines are safely collected, while larger particles are blocked by mesh grates covering the bottoms of the feet to avoid clogging the system. During locomotion, the vacuum pressure is monitored by external pressure sensors and regulated by computer-controlled solenoid valves, both located in the support system. 
HeartLander achieves inchworm-like locomotion by coordinating prehension cycles and wire-actuated translations between the two modules of the drive mechanism. The distance and orientation between the two modules are controlled by three superelastic nitinol wires. The wires are linearly actuated by electric motor-driven belts in the support system. They pass freely through the tether and rear body section, and are attached distally to the front body section. The translations of the wires by the motors are transmitted to the modules at the drive mechanism by three plastic sheaths that enclose the wires in the tether. The sheaths are attached proximally to the stationary motor stage, and distally to the rear body section. The super-elasticity of nitinol allows the wires to support tension and compression (i.e. pulling and pushing) without permanently deforming. This eliminates the need for shape-restoring components (like springs) that are required in cable-drive transmissions. Locomotion is a cyclic process, one cycle of which is schematically illustrated in Fig. 3(a). The front module is advanced by pushing on the wires while the rear module has active suction. Retraction of the rear module to the advanced front module is accomplished by pulling back on the wires while the front module has active suction. Although the configuration of the sheaths and enclosed wires is unconstrained during locomotion, some slack must be maintained between the support system and the rear module in order for this locomotion scheme to work (i.e. the tether must not be taut). Turning is achieved by differentially changing the lengths of the side wires, illustrated in Fig. 3(b). The wires pass through triads of eyelets attached to a support spring between the front and rear body sections. This prevents the wires from bowing outward during turning and ensures that the wires maintain equal distances from one another (Fig. 3(c)). The support spring has a very low spring constant $(\mathrm{k}=0.012 \mathrm{~N} / \mathrm{mm})$, thus the restoring force is negligible as compared to that of the wires. The three independently actuated wires provide three degrees of freedom (DOF) between the modules, two angular and one translational. The two angular DOF allow the device to adapt to the curvature of the heart (i.e. pitch) as well as turn laterally (i.e. yaw).

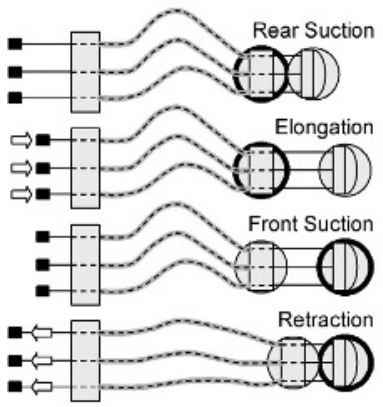

(a)

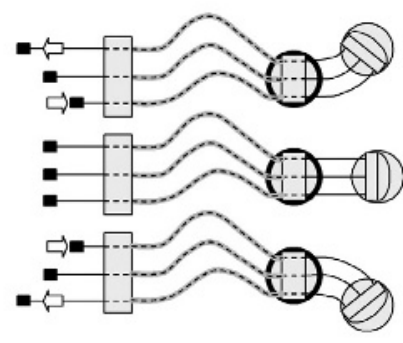

(b)

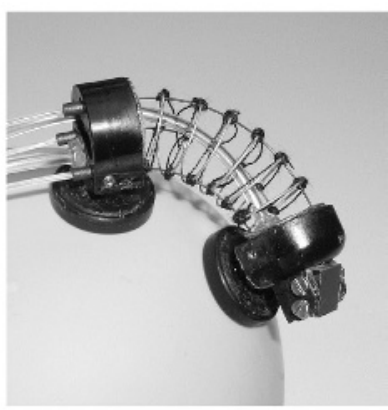

(c)

Fig. 3. (a) Illustration of the locomotion cycle of the wire-actuated HeartLander prototype (dark ring indicates the module that has active suction at each step of the process). (b) Illustration of steering. (c) HeartLander prototype with spring to allow sharp turns without bowing of the wires $\left(90^{\circ}\right.$ shown here $) .3 \mathrm{DOF}$ allow the device to conform to surface curvature 
The locomotion of the device and operation of the surgical end-effector are controlled by the physician using a PC-based graphical user interface that provides video feedback. A joystick controls the direction of travel and offers two speeds of travel (shown in Fig. 6). The previously described mechanical details of the locomotive process are handled by the computer, and thus are transparent to the user. Visual feedback from the front module is relayed to an external video camera by a $1.6-\mathrm{mm}$ diameter fiber optic endoscope running through the tether, and displayed to the user on the monitor. A mirror is mounted to the front module in order to angle the view of the scope toward the surface of the heart. Along with a channel for the scope, both modules contain a 3-mm working port through which tools can be deployed for a variety of epicardial interventions.

\section{Testing}

\subsection{Prehensile Testing with a Poultry Model}

The support system pump provides a vacuum pressure of $-0.08 \mathrm{~N} / \mathrm{mm}^{2}$, which was found to be effective and safe for use in FDA-approved cardiac stabilizers. Based on this pressure, the normal and tangential forces calculated to dislodge one of the modules are $1.76 \mathrm{~N}$ and $0.87 \mathrm{~N}$, respectively. Bench testing using a force gauge to dislodge the device from a poultry model verified normal and tangential forces of $2.01 \mathrm{~N}$ and $0.86 \mathrm{~N}$. The tangential force that can be resisted by the device will be increased significantly by reducing the profile of the next design.

\subsection{Locomotion on the Beating Heart Using a Porcine Model}

The wire-actuated HeartLander prototype was tested on three open-chest, beatingheart porcine models. The pericardium had been removed entirely and HeartLander was placed directly on the epicardium by hand. The device was able to maintain prehension on the exposed epicardium without being dislodged by the natural beating motion of the heart. Video recorded from the device was displayed for the surgeon on the computer monitor (shown in Fig. 4). Locomotion was also captured on a handheld video camera as the device traveled approximately $50 \mathrm{~mm}$ across the epicardium from the left ventricle to the right ventricle, crossing the left anterior descending coronary artery (LADA), as shown in Fig. 5. A cardiac surgeon verified that no damage was done to the epicardium as a result of the prehension or locomotion.

\section{Discussion}

The results presented herein demonstrate the feasibility of adhering to and maneuvering on the epicardium of a beating heart using the HeartLander prototype. Future 
porcine tests will proceed from open-heart testing to minimally invasive testing using a subxiphoid approach.

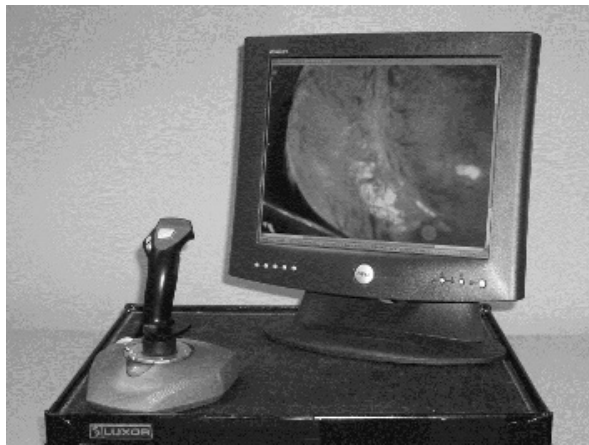

(a)

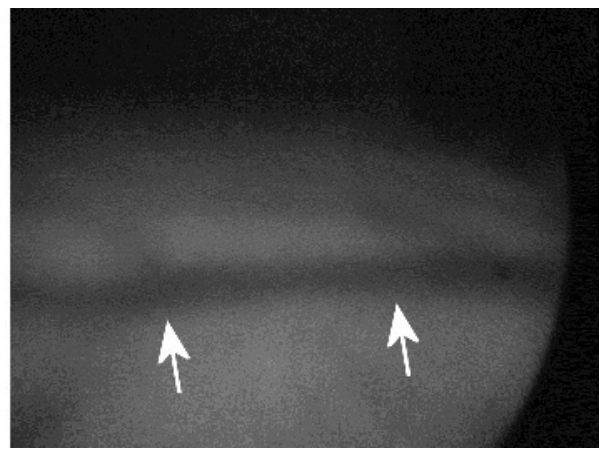

(b)

Fig. 4. (a) The HeartLander control interface: joystick for control of locomotion, and monitor to display video from the device camera. (b) View of the left anterior descending artery (LADA) through the device camera (arrows highlight LADA)
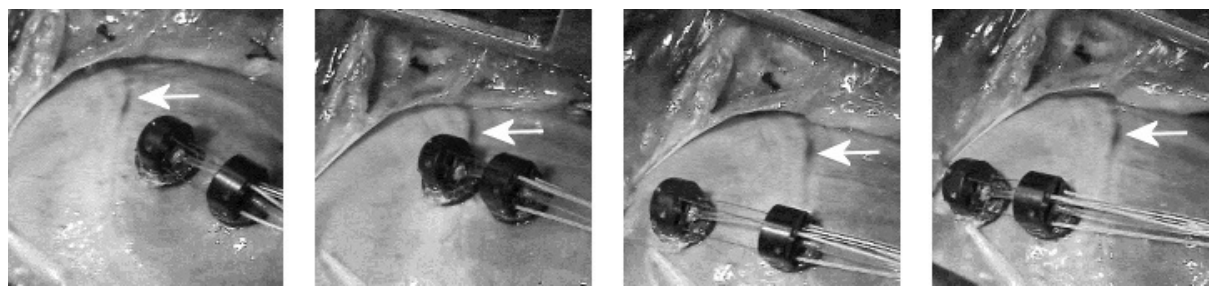

Fig. 5. A time sequence of photographs showing the HeartLander prototype crossing the left anterior descending artery (LADA) on a beating porcine heart (arrows highlight a reference point along the LADA for comparison between frames)

By employing a modular design for end-effector attachment, HeartLander will be capable of performing a variety of surgical treatments. The first application planned for evaluation is epicardial lead placement for resynchronization [9]. The actuation for the end-effectors will either be provided directly by an on-board motor or transmitted from an external motor through the tether.

As the research continues, we plan to develop end-effectors for HeartLander for more innovative procedures, such as epicardial delivery of myoblasts or stem cells for regeneration of the failing myocardium. Ultimately, we envision adoption of HeartLander-based intrapericardial therapies not only by minimally invasive cardiac surgeons, but also by interventional cardiologists and electrophysiologists $[14,15]$. 
Acknowledgments. Funding provided by the Simeon M. Jones, Jr. and Katharine Reed Jones Fund and the John D. Schaub's Sons Memorial Fund of the The Pittsburgh Foundation.

\section{References}

1. Mack MJ (2001) Minimally invasive and robotic surgery. JAMA 285:568-572.

2. Falk V, Diegler A, Walther T, Autschbach R, Mohr FW (2000) Developments in robotic cardiac surgery. Curr Opin Cardiol 15:378-387.

3. Borst C, Gründeman PF (1999): Minimally invasive coronary artery bypass grafting: an experimental perspective. Circulation 99:1400-1403.

4. Zenati, MA (2001) Robotic heart surgery. Cardiol Rev 9:287-294.

5. Gründeman PF, Budde R, Beck HM, van Boven W-J, Borst C (2003) Endoscopic exposure and stabilization of posterior and inferior branches using the endo-starfish cardiac positioner and the endo-octopus stabilizer for closed-chest beating heart multivessel CABG: hemodynamic changes in the pig. Circulation 108:11-34.

6. Çavuşoğlu MC, Williams W, Tendick F, Sastry SS (2003) Robotics for telesurgery: second generation Berkeley/UCSF laparoscopic telesurgical workstation and looking towards the future applications. Industrial Robot 30(1):22-29.

7. Li R-K, Jia Z-Q, Weisel RD, Merante F, Mickle DAG (1999) Smooth muscle cell transplantation myocardial scar tissue improves heart function. J Mol Cell Cardiol 31:513522.

8. Losordo DW, Vale PR, Isner JM (1999) Gene therapy for myocardial angiogenesis. Am Heart J 138(2) Pt 2: S132-41.

9. Leclercq C, Kass DA (2002) Retiming the failing heart: principles and current clinical status of cardiac resynchronization. J Am Coll Cardiol 39:194-201.

10. Lee R, Nitta T, Schuessler RB, Johnson DC, Boineau JP, Cox JL (1999) The closed heart MAZE: A nonbypass surgical technique. Ann Thorac Surg 2:1696-1702.

11. Gleason JD, Nguyen KP, Kissinger KV, Manning WJ, Verrier RL (2002) Myocardial drug distribution pattern following intrapericardial delivery: an MRI analysis. J Cardiovasc Magn Reson 4(3): 311-316.

12. Boekstegers P, Raake P, Al Ghobainy R, Horstkotte J, Hinkel R, Sandner T, Wichels R, Meisner F, Thein E, March K, Boehm D, Reichenspurner H (2002) Stent-based approach for ventricle-to-coronary artery bypass. Circulation 106:1000-1006.

13. Siegel M, Gunatilake P, Podnar G (1998) Robotic assistants for aircraft inspectors. Instrumentation Measurement Mag, 1(1):16-30.

14. Schweikert RA, Saliba WI, Tomassoni G, Marrouche NF, Cole CR, Dresing TJ, Tchou PJ, Bash, D, Beheiry S, Lam C, Kanagaratna L, Natale A (2003) Percutaneous pericardial instrumentation for endo-epicardial mapping of previously failed ablations. Circulation 108:1329-1335.

15. Sosa E, Scanavacca M, D'Avila A, Oliveira F, Ramires JAF (2000) Nonsurgical transthoracic epicardial catheter ablation to treat recurrent ventricular tachycardia occurring late after myocardial infarction. J Am Coll Cardiol 35:1442-1449. 